\title{
Association between exposure to antimicrobial household products and allergic symptoms
}

\author{
Soyoung Hong ${ }^{1,2}$, Ho-Jang Kwon ${ }^{3}$, Won-Jun Choi ${ }^{4}$, Wan Ryung Lim ${ }^{1}$, Jeonghoon Kim ${ }^{1,2}$, \\ KyooSang $\mathrm{Kim}^{1}$ \\ ${ }^{1}$ Department of Environmental Health Research, Seoul Medical Center, Seoul; ${ }^{2}$ Graduate School of Public Health, Seoul \\ National University, Seoul; ${ }^{3}$ Department of Preventive Medicine, Dankook University College of Medicine, Cheonan; \\ ${ }^{4}$ Department of Occupational \& Environmental Medicine, Gachon University Gil Medical Center, Incheon, Korea
}

Objectives Antimicrobial chemicals are used in a variety of household and personal care products. Exposure to antimicrobial household products has been hypothesized to lead to allergic diseases in children.

Methods We investigated antimicrobial household product exposure and allergic symptoms in Korean children. An antimicrobial exposure (AE) score was derived. To examine the symptoms of allergic diseases (current wheeze, current rhinitis, and current eczema) in the past 12 months, we used a questionnaire based on the core module of the International Study of Asthma and Allergies in Children. Complete data for the analysis were available for 25,805 of the 35,590 (72.5\%) children.

Results The prevalence of current allergic diseases was as follows: wheeze, 5.6\%; allergic rhinitis, 32.6\%; and eczema, $17.7 \%$. The mean (standard deviation) AE score was 14.3 (9.3) (range: 0-40). Compared with subjects with a low AE score (reference), subjects with a high AE score (fourth quartile) were more likely to have symptoms of wheezing and allergic rhinitis (adjusted odds ratio [aOR] for wheezing 1.24, 95\% confidence interval $[\mathrm{Cl}], 1.05-1.45, p$ for trend=0.24; aOR for allergic rhinitis 1.30, $95 \% \mathrm{Cl}, 1.20-1.4 \mathrm{O}$, $p<0.01)$.

Conclusions These findings suggest that frequent use of antimicrobial household products was associated with current wheeze and current allergic rhinitis.

Keywords Allergic diseases, Allergic rhinitis, Antimicrobial, Asthma, Children, Triclosan

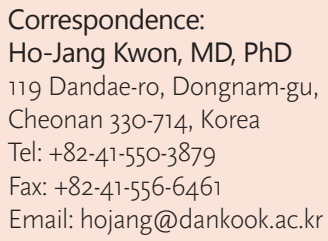

Received: July 9, 2014

Accepted: October 2, 2014

Published online: November 21, 2014

This article is available from: http://e-eht.org/

\section{Introduction}

Antimicrobial agents generally refer to products or ingredients that kill microorganisms or inhibit the organisms' growth. Antimicrobial chemicals are used in a variety of household and personal care products, such as detergents, food storage containers, toothpastes, mouthwash, deodorants, soap, toys, polymers, and textile fibers $[1,2]$. The typical ingredients of antibacterial products are triclosan and triclocarban. Several studies have demonstrated that products containing triclosan were no more effective at eliminating bacteria than soap and water [3]. In laboratory studies, these products have also been shown to disrupt hor- mones and to encourage the growth of drug-resistant bacteria $[4,5]$.

Although there have been increasing concerns about the use of antimicrobial household products, the ingredients of household products have not been investigated thoroughly. A previous study identified antimicrobial agents such as triclosan and triclocarban in marketed soaps, toothpastes and laundry detergents, especially the ones that were specifically labelled 'antimicrobial products' [6]. Antimicrobial household products are widely distributed in the market. Due to extensive use of such products, there is the potential for people of all ages to experience lifetime exposure. Given the duration, especially in child- 
hood, of the antimicrobial chemical exposure, and the potential health effects, this issue needs attention. Antimicrobial chemicals have been detected in human samples (urine, breast milk, and serum) [7-9]. However, the potential risks of antimicrobial chemical exposure have not been well described. The US Food and Drug Administration has nominated antibacterial chemicals to the National Toxicology Program for toxicological evaluations [10].

Previous studies on the health effects of antibacterial chemicals were performed either in vitro [11] or in occupational settings $[12,13]$. Although there are previous studies that report chemical exposure increases the risk of allergic symptoms in children, they were generally focused on a relatively broad spectrum of chemical products $[14,15]$ or some specific chemicals such as propylene glycol and glycol ethers [16], rather than on antimicrobial household products.

In this study, we investigated the association between exposure to antimicrobial products and allergic symptoms in children.

\section{Materials and Methods}

\section{Study Population}

Subjects were recruited from the 'Seoul Atopy Friendly School' cross-sectional survey from May to October 2012 [16]. The eligible participants were 58,117 children in 423 facilities. The parents or guardians of the subjects provided written informed consent and decided voluntarily whether to participate. Among these subjects, 35,590 answered a questionnaire (response rate of $61.2 \%$ ). Those subjects who did not answer one or more of the following survey questions were excluded: omitted individual information, such as age or parental history of allergic diseases $(n=2,707)$; omitted the history of allergic symptoms $(\mathrm{n}=1,423)$; and omitted the frequency of antimicrobial product use $(n=5,655)$. Ultimately, 25,805 subjects were included in the analysis. The study procedure was reviewed and approved by the Ethical Committee of Seoul Medical Center.

\section{Antimicrobial Product Use in the Household}

Using a questionnaire, we asked about the frequency of the use of 10 selected products labelled 'antimicrobial' or 'antibacterial': toothpastes, soaps, hand sanitizers, dishwashing detergents, fungicides, laundry detergents, deodorants, aerosol cleaners, wet wipes, and household pesticides. For each product, the available responses for frequency were as follows: not at all, less than once per week, approximately once per week, more than once per week, and nearly every day. Frequency was then converted into a score: 0 for not at all, 1 for less than once per week, 2 for approximately once per week, 3 for more than once per week, and 4 for nearly every day. The sum of the points was considered to be a surrogate for the antimicrobial exposure (AE) score in the household.

\section{Status of Allergic Diseases}

Questions regarding the status of allergic diseases were derived from the International Study of Asthma and Allergies in Childhood (ISAAC) core module [17]. Similar questions have been described elsewhere [18]. Briefly, using the official Korean version of the ISAAC questionnaire, we determined the prevalence of wheeze, rhinitis, and eczema in the past 12 months (referred to as current wheeze, current rhinitis, and current eczema, respectively). The following questions were asked: 1) Has your child had wheezing or whistling in the chest in the past 12 months? (Current wheeze); 2) In the past 12 months, has your child had a problem with sneezing or a runny or blocked nose when he/she did not have a cold or the flu? (Current rhinitis); and 3) Has your child had an itchy rash at any time in the past 12 months? (Current eczema).

\section{Statistics}

One-way analysis of variance (ANOVA) and $t$-tests were performed to compare mean $\mathrm{AE}$ scores. Logistic regression analyses were performed to calculate the odds ratios (ORs) and 95\% confidence intervals (CIs) of AE scores for each allergic disease symptom. In the logistic regression models, the $\mathrm{AE}$ score was treated as a categorical variable and grouped into quartiles. In the multiple logistic regression models, gender, age (as a continuous variable), second-hand smoke exposure (i.e., Is your child exposed to second hand smoke at least once a day? Yes or No), and a parental history of allergic diseases (i.e., Have you ever been diagnosed with allergic diseases by a doctor? Yes or No) were considered to be covariates. The results were considered statistically significant for $p$-values $<0.05$. All of the statistics were performed using STATA version 10.0 (StataCorp, College Station, TX, USA).

\section{Results}

\section{Participant Characteristics}

The demographic and general characteristics of the subjects are presented in Table 1 . There were 13,013 boys (50.4\%) and 12,792 girls (49.6\%). The mean age of the subjects was 7.0 
(standard deviation [SD], 3.0; range, 0-13). Of these, 5,212 (20.2\%) subjects had been exposed to second-hand smoke. The prevalence of current wheeze, current rhinitis, and current eczema was $5.6 \%, 32.6 \%$, and $17.7 \%$, respectively. The mean $\mathrm{AE}$ score was 14.3 (SD, 9.3; range, 0-40).

\section{Frequency of Antimicrobial Household Product use}

Table 2 shows the frequency of antimicrobial product use. Certain products were used frequently in the household, whereas other products, such as fungicide, pesticides, and aerosol cleaners, were used infrequently. For example, a response of using specific products nearly every day was indicated for the following: dishwashing detergent, $41.1 \%$; soap, 39.3\%; hand sanitizer, $36.7 \%$; toothpaste, $33.3 \%$; and wet wipes, $30.2 \%$. Only $5.1 \%$ and $2.0 \%$ of participants indicated that they used fungicide and pesticide, respectively, nearly every day.

\section{Antimicrobial Product Exposure}

$\mathrm{AE}$ scores were compared by the characteristics of the subjects (Table 3). The score was not significantly different between

Table 1. The characteristics of 25,805 participants

\begin{tabular}{llrr}
\hline Characteristics & & $\mathrm{n}$ & $\%$ \\
\hline Gender & Boys & 13,013 & 50.4 \\
& Girls & 12,792 & 49.6 \\
Age (yr) & $0-2$ & 3,764 & 14.6 \\
& $3-5$ & 7,828 & 30.3 \\
& $6-8$ & 7,780 & 30.2 \\
Second-hand smoke & $9-13$ & 6,433 & 24.9 \\
exposure & Yes & 5,212 & 20.2 \\
Parental history of allergic & No & 20,593 & 79.8 \\
disease & No & 10,212 & 39.6 \\
Allergic symptoms & Current wheeze & 15,593 & 60.4 \\
& Current rhinitis & 8,435 & 5.6 \\
Antimicrobial exposure score & Mean (SD) & 14.3 & 32.6 \\
\hline
\end{tabular}

boys and girls. The $\mathrm{AE}$ score was inversely associated with age $(p<0.01)$. A significantly higher score was observed with exposure to second-hand smoke $(p<0.01)$ and a parental history of allergic diseases $(p<0.01)$. The AE score of those subjects who had current wheeze was significantly higher than the score of subjects who had not experienced wheeze in the past 12 months (mean 15.3 and 14.3, respectively; $p<0.01$ ). Similar results were observed for current rhinitis and current eczema.

\section{Association between the AE Score and Allergic Symptoms}

The results of logistic regression analyses are presented in Table 4. For current wheeze, the unadjusted OR was 1.45 (95\% $\mathrm{CI}=1.24-1.70)$ and the adjusted $\mathrm{OR}$ was $1.24(95 \% \mathrm{CI}=1.05$ 1.70) for the highest quartile of score compared with the reference group. For current rhinitis, the unadjusted OR was 1.34 $(95 \% \mathrm{CI}=1.24-1.45)$ and the adjusted OR was $1.30(95 \%$

Table 3. Antimicrobial exposure score according to the characteristics of the subjects

\begin{tabular}{llccc}
\hline Characteristics & & Mean & SD & $p$-value \\
\hline Gender & Boys & 14.2 & 9.3 & 0.94 \\
& Girls & 14.4 & 9.3 & \\
Age (yr) & $0-2$ & 15.3 & 9.2 & $<0.01$ \\
& $3-5$ & 14.9 & 9.2 & \\
& $6-8$ & 14.1 & 9.3 & \\
Second-hand smoke exposure & $9-13$ & 13.2 & 9.4 & \\
Pes & 14.9 & 9.4 & $<0.01$ \\
Parental history of allergic & No & 14.2 & 9.2 & \\
diseases & Yes & 15.0 & 9.2 & $<0.01$ \\
Current wheeze & No & 13.9 & 9.3 & \\
& Yes & 15.3 & 9.3 & $<0.01$ \\
Current rhinitis & No & 14.3 & 9.3 & \\
\multirow{2}{*}{ Current eczema } & Yes & 14.9 & 9.2 & $<0.01$ \\
& No & 14.3 & 9.3 & \\
& Yes & 14.7 & 9.2 & $<0.01$ \\
\hline
\end{tabular}

${ }^{a} p$-value from $t$-test and ANOVA.

Table 2. Frequency of the use of antimicrobial household products

\begin{tabular}{|c|c|c|c|c|c|}
\hline Category & No use & Less than one time per week & About once per week & More than once per week & Nearly every day \\
\hline Toothpaste & $15,545(60.2)$ & $788(3.1)$ & $311(1.2)$ & 566 (2.2) & $8,595(33.3)$ \\
\hline Soap & $12,288(47.6)$ & $1,347(5.2)$ & $634(2.5)$ & $1,388(5.4)$ & $10,148(39.3)$ \\
\hline Hand sanitizer & $10,103(39.1)$ & $2,426(9.4)$ & $1,251(4.9)$ & $2,543(9.9)$ & $9,482(36.7)$ \\
\hline Dishwashing detergent & 7,976 (30.9) & $3,274(12.7)$ & $2,082(8.0)$ & $1,876(7.3)$ & $10,597(41.1)$ \\
\hline Fungicide & $14,058(54.5)$ & 6,801 (26.4) & $3,186(12.3)$ & $1,252(4.8)$ & $508(2.0)$ \\
\hline Laundry detergent & $10,661(41.3)$ & $2,111(8.2)$ & $2,322(9.0)$ & $5,591(21.7)$ & $5,120(19.8)$ \\
\hline Deodorant & $16,566(64.2)$ & $3,907(15.1)$ & $1,933(7.5)$ & $1,694(6.6)$ & $1,705(6.6)$ \\
\hline Aerosol cleaners & $12,358(47.9)$ & $6,455(25.0)$ & $3,884(15.0)$ & $2,062(8.0)$ & $1,046(4.1)$ \\
\hline Wet wipes & $8,778(34.0)$ & $3,342(13.0)$ & $1,993(7.7)$ & $3,902(15.1)$ & $7,790(30.2)$ \\
\hline Household pesticides & $14,756(57.2)$ & $5,513(21.3)$ & $1,955(7.6)$ & $2,267(8.8)$ & $1,314(5.1)$ \\
\hline
\end{tabular}

Values are presented as number (\%). 
Table 4. Crude odds ratio (OR) and adjusted odds ratio (aOR) for asthma, allergic rhinitis, and eczema according to the quartile of the antimicrobial exposure score

\begin{tabular}{|c|c|c|c|c|c|c|}
\hline & & n (\% cases) & Unadjusted OR & $95 \% \mathrm{Cl}$ & $\mathrm{aOO} \mathrm{R}^{\mathrm{a}}$ & $95 \% \mathrm{Cl}$ \\
\hline \multicolumn{7}{|c|}{ Current wheeze } \\
\hline \multirow[t]{5}{*}{ Quartiles } & $1 \mathrm{st}$ & $290(4.4)$ & 1.00 & & 1.00 & \\
\hline & 2nd & $413(5.8)$ & 1.32 & $1.13,1.54$ & 1.17 & $1.03,1.37$ \\
\hline & $3 r d$ & 365 (5.8) & 1.33 & $1.13,1.55$ & 1.19 & $1.02,1.40$ \\
\hline & 4th & 367 (6.3) & 1.45 & $1.24,1.70$ & 1.24 & $1.05,1.45$ \\
\hline & $p$ for trend & & $<0.01$ & & 0.02 & \\
\hline \multicolumn{7}{|c|}{ Current rhinitis } \\
\hline \multirow[t]{5}{*}{ Quartiles } & 1 st & $1,888(28.9)$ & 1.00 & & 1.00 & \\
\hline & 2nd & $2,381(33.3)$ & 1.23 & $1.14,1.32$ & 1.20 & $1.11,1.29$ \\
\hline & $3 r d$ & $2,090(33.2)$ & 1.22 & $1.14,1.32$ & 1.19 & $1.10,1.28$ \\
\hline & 4th & $2,055(35.3)$ & 1.34 & $1.24,1.45$ & 1.30 & $1.20,1.40$ \\
\hline & $p$ for trend & & $<0.01$ & & $<0.01$ & \\
\hline \multicolumn{7}{|c|}{ Current eczema } \\
\hline \multirow[t]{5}{*}{ Quartiles } & $1 \mathrm{st}$ & 1,067 (16.3) & 1.00 & & 1.00 & \\
\hline & 2nd & $1,294(18.1)$ & 1.13 & $1.04,1.24$ & 1.07 & $0.98,1.17$ \\
\hline & $3 \mathrm{rd}$ & $1,151(18.3)$ & 1.15 & $1.05,1.26$ & 1.08 & $0.94,1.19$ \\
\hline & 4th & $1,053(18.1)$ & 1.13 & $1.03,1.24$ & 1.04 & $0.94,1.14$ \\
\hline & $p$ for trend & & 0.01 & & 0.44 & \\
\hline
\end{tabular}

$\mathrm{Cl}$, confidence interval.

${ }^{a}$ Adjusted for age, gender, second-hand smoke exposure, and a parental history of allergic diseases.

$\mathrm{CI}=1.20-1.40)$ for the highest quartile compared with the reference group. For current eczema, the unadjusted OR was 1.13 $(95 \% \mathrm{CI}=1.03-1.24)$ and the adjusted $\mathrm{OR}$ was 1.04 (95\% CI= 0.94-1.14) for the highest quartile compared with the reference group. The OR of current wheeze increased with the quartiles of the $\mathrm{AE}$ score ( $p$ for trend $<0.01$ ). The association was still significant after adjustment for age, gender, second-hand smoke exposure, and a parental history of allergic diseases ( $p$ for trend $0.02)$. Similar results were observed for current rhinitis. The association between the antimicrobial product exposure level and current eczema was no longer significant after adjustment ( $p$ for trend 0.44).

\section{Discussion}

A dose-dependent relationship was noted between AE scores and allergic symptoms. For current wheeze and current rhinitis, the association was significant after adjustment for potential confounding variables. After the adjustment, the association between current eczema and AE score disappeared. These findings suggest that frequent use of antimicrobial household products was associated with current wheeze and current allergic rhinitis.

Various types of antimicrobial ingredients such as triclosan and triclocarban are used in consumer products [19]. Triclosan is one of the most well-known antibacterial and antifungal agents that has been in use since the early 1970s. Although each species of bacterium has unique features, including specific enzymes and structural characteristics, triclosan has broad anti- bacterial specificity. Thus, triclosan is usually included in personal care products, including soaps, deodorants, toothpastes, and mouthwashes $[20,21]$. For example, a marketplace study of the US showed that nearly half of all commercial soaps contained triclosan (76\% of liquid soaps and $29 \%$ of bar soaps, a total of $45.5 \%$ of all soaps investigated) [22]. The popularity of antibacterial consumer products has led to increased consumer use of triclosan [22,23].

Triclosan and its metabolites were omnipresent in the analyzed plasma and milk of 36 Swedish nursing mothers [9]. The concentrations were higher in both the plasma and milk from mothers who used personal care products containing triclosan than in the plasma and milk from mothers who did not. This result suggested that personal care products were a main source of exposure to triclosan.

Previous studies reported that the use of household chemical products in the perinatal period was associated with persistent wheeze and abnormal lung function in children $[13,14]$. This finding is consistent with the results of our study, in which the use of household antimicrobial products was associated with symptoms of allergic diseases in children, although the agents of interest were not the same. Bertelsen et al. [24] reported that urinary triclosan was associated with allergic sensitization in Norwegian children. They found that the risk of current rhinitis significantly increased with increasing urinary concentrations of triclosan. Using data from the National Health and Nutrition Examination Survey, Clayton et al. [25] reported that a higher concentration of urinary triclosan was associated with increased 
risk of allergies or hay fever.

In addition to triclosan, antimicrobial household products contain other antibacterial agents. Chloroxylenol, also known as $p$-chloroxylenol, is a compound that has been used as an active preservative agent in antimicrobial products. Chloroxylenol is used in nail treatments, after-sun products, eye shadows, shampoos, foot moisturizers, and hand creams, for antimicrobial, deodorant, and preservative purposes. Chloroxylenol use has been associated with contact dermatitis [26,27]. Tetrasodium ethylenediaminetetraacetate (EDTA) is mainly used to sequester metal ions in aqueous solution. Many shampoos, body washes, conditioners, moisturizers, sunscreens, and body powder products include this agent. A relationship between tetrasodium EDTA and occupational asthma and contact dermatitis has been reported [28]. Triclocarban serves the purpose of a cosmetic biocide, deodorant agent, and preservative and is mainly used as an ingredient in antimicrobial household products. Triclocarban is also used in soap, facial cleansers, and acne treatments. Although triclocarban itself does not seem to induce skin sensitization or irritation, concerns about the increased risk of allergic diseases through the increased use of antibacterial products (i.e., the hygiene hypothesis) still remain.

The hygiene hypothesis is a possible explanation for the increased risk of allergic diseases with the use of antimicrobial household products. Previous studies reported that infections in childhood are associated with a reduction in the incidence of allergic diseases $[29,30]$. According to the main concept underlying the hygiene hypothesis, exposure to commensal microorganisms is essential to appropriately stimulate Thelper 1 (TH1) and T helper 2 (TH2) cells. Appropriate stimulation of TH1 and $\mathrm{TH} 2$ plays an important role in the maturation of the immune system [31]. In most children with allergic diseases, TH1 and $\mathrm{TH} 2$ are not balanced, as there are more $\mathrm{TH} 2$ cells that promote antibody production. This imbalance may result in increased risks of autoimmune reactions and allergic diseases. Studies have reported that an excessively clean childhood can reduce the chance of infection in growing children but increase the risk of allergic diseases. Antimicrobial agents may be implicated in the hygiene hypothesis because antimicrobial products kill bacteria in the household, resulting in an excessively clean environment.

Reverse causality cannot be discounted in the relationship between exposure to antimicrobial products and allergic symptoms. It is possible that the status of the allergic symptoms affects the frequency of antimicrobial product use. Unfortunately, in our study, we cannot determine the temporal relationship between the use of antimicrobial products and the allergic symptoms due to the cross-sectional study design. It is possible that people who have allergic symptoms use antimicrobial household products more frequently. In the present study, the AE score was higher if parents had a history of allergic diseases. However, we adjusted for this possible confounder in the analyses.

Not all personal care products contain antimicrobial ingredients. We asked the subjects about the use of products labelled 'antimicrobial' or 'antibacterial' to assess the exposure to antimicrobial products. There may have been misclassification of exposure if products that were not labelled as 'antimicrobial' or 'antibacterial' contained the chemicals of interest. However, according to a report on a recent market survey, antimicrobial agents such as triclosan and triclocarban were not detected in products that were not labelled as 'antimicrobial' or 'antibacterial. [6] Although most antimicrobial products contain antimicrobial chemicals, the use of antimicrobial agents in consumer products has not been studied extensively [22]. Further investigations are needed to reveal the specific ingredient of each product and to study the mechanism of the allergic symptoms caused by these antimicrobial products.

Our study may not have taken into account all of the possible confounders. Knowledge and information about the health effects of antimicrobial agents may have affected the choice of household products used. The education level of the parents may have been associated with attitudes toward household chemical products. Unfortunately, these factors were not measured in our study.

The present study has several advantages. This investigation is a large population-based study that included children of a wide range of ages. Previous studies have mainly focused on the health effects of triclosan in adults. We focused on the health effects of antimicrobial products that are widely used at home. Using a semiquantitative questionnaire meant that we could save resources and gather more information on various variables that affect allergic diseases.

In summary, the present study has shown an association between an increased risk of allergic symptoms and antimicrobial household product exposure. This research has emphasized the need for long-term longitudinal studies to confirm the association and to clarify the pathophysiologic mechanisms involved.

\section{Acknowledgements}

The authors thank Seoul Metropolitan Government and 25 Public Health Centers.

\section{Conflict of Interest}

The authors have no conflicts of interest with material presented in this paper. 


\section{References}

1. Bhargava HN, Leonard PA. Triclosan: applications and safety. Am J Infect Control 1996;24(3):209-218.

2. McBain AJ, Bartolo RG, Catrenich CE, Charbonneau D, Ledder RG, Price BB, et al. Exposure of sink drain microcosms to triclosan: population dynamics and antimicrobial susceptibility. Appl Environ Microbiol 2003;69(9):5433-5442.

3. Aiello AE, Larson EL, Levy SB. Consumer antibacterial soaps: effective or just risky? Clin Infect Dis 2007;45 Suppl 2:S137-S147.

4. Crofton KM, Paul KB, Devito MJ, Hedge JM. Short-term in vivo exposure to the water contaminant triclosan: evidence for disruption of thyroxine. Environ Toxicol Pharmacol 2007;24(2):194-197.

5. Aiello AE, Marshall B, Levy SB, Della-Latta P, Lin SX, Larson E. Antibacterial cleaning products and drug resistance. Emerg Infect Dis 2005;11(10): 1565-1570.

6. Seoul Metropolitan Government. Report on the prevention and management of environmental diseases. Seoul: Metropolitan Government; 2013, p. 412-414 (Korean).

7. Li X, Ying GG, Zhao JL, Chen ZF, Lai HJ, Su HC. 4-Nonylphenol, bisphenol-A and triclosan levels in human urine of children and students in China, and the effects of drinking these bottled materials on the levels. Environ Int 2013;52:81-86.

8. Bedoux G, Roig B, Thomas O, Dupont V, Le Bot B. Occurrence and toxicity of antimicrobial triclosan and by-products in the environment. Environ Sci Pollut Res Int 2012;19(4):1044-1065.

9. Allmyr M, Adolfsson-Erici M, McLachlan MS, Sandborgh-Englund G. Triclosan in plasma and milk from Swedish nursing mothers and their exposure via personal care products. Sci Total Environ 2006;372(1):87-93.

10. Fang JL, Stingley RL, Beland FA, Harrouk W, Lumpkins DL, Howard P. Occurrence, efficacy, metabolism, and toxicity of triclosan. J Environ Sci Health C Environ Carcinog Ecotoxicol Rev 2010; 28(3):147-171.

11. Witorsch RJ, Thomas JA. Personal care products and endocrine disruption: a critical review of the literature. Crit Rev Toxicol 2010; 40 Suppl 3:1-30.

12. Lindström I, Suojalehto H, Henriks-Eckerman ML, Suuronen K. Occupational asthma and rhinitis caused by cyanoacrylate-based eyelash extension glues. Occup Med (Lond) 2013;63(4):294-297.

13. Quirce S, Barranco P. Cleaning agents and asthma. J Investig Allergol Clin Immunol 2010;20(7):542-550.

14. Henderson J, Sherriff A, Farrow A, Ayres JG. Household chemicals, persistent wheezing and lung function: effect modification by atopy? Eur Respir J 2008;31(3):547-554.

15. Sherriff A, Farrow A, Golding J, Henderson J. Frequent use of chemical household products is associated with persistent wheezing in pre-school age children. Thorax 2005;60(1):45-49.

16. Choi H, Schmidbauer N, Sundell J, Hasselgren M, Spengler J,
Bornehag CG. Common household chemicals and the allergy risks in pre-school age children. PLoS One 2010;5(10):e13423.

17. Ellwood P, Asher MI, Beasley R, Clayton TO, Stewart AW; ISAAC Steering Committee. The international study of asthma and allergies in childhood (ISAAC): phase three rationale and methods. Int J Tuberc Lung Dis 2005;9(1):10-16.

18. Hong S, Son DK, Lim WR, Kim SH, Kim H, Yum HY, et al. The prevalence of atopic dermatitis, asthma, and allergic rhinitis and the comorbidity of allergic diseases in children. Environ Health Toxicol 2012;27:e2012006.

19. Tan L, Nielsen NH, Young DC, Trizna Z; Council on Scientific Affairs, American Medical Association. Use of antimicrobial agents in consumer products. Arch Dermatol 2002;138(8):1082-1086.

20. Thompson A, Griffin P, Stuetz R, Cartmell E. The fate and removal of triclosan during wastewater treatment. Water Environ Res 2005;77(1):63-67.

21. McAvoy DC, Schatowitz B, Jacob M, Hauk A, Eckhoff WS. Measurement of triclosan in wastewater treatment systems. Environ Toxicol Chem 2002;21(7):1323-1329.

22. Perencevich EN, Wong MT, Harris AD. National and regional assessment of the antibacterial soap market: a step toward determining the impact of prevalent antibacterial soaps. Am J Infect Control 2001;29(5):281-283.

23. Adolfsson-Erici M, Pettersson M, Parkkonen J, Sturve J. Triclosan, a commonly used bactericide found in human milk and in the aquatic environment in Sweden. Chemosphere 2002;46(9-10): 1485-1489.

24. Bertelsen RJ, Longnecker MP, Løvik M, Calafat AM, Carlsen KH, London SJ, et al. Triclosan exposure and allergic sensitization in Norwegian children. Allergy 2013;68(1):84-91.

25. Clayton EM, Todd M, Dowd JB, Aiello AE. The impact of bisphenol $\mathrm{A}$ and triclosan on immune parameters in the U.S. population, NHANES 2003-2006. Environ Health Perspect 2011;119(3):390396.

26. Ye X, Zhou X, Furr J, Ahn KC, Hammock BD, Gray EL, et al. Biomarkers of exposure to triclocarban in urine and serum. Toxicology 2011;286(1-3):69-74.

27. Berthelot C, Zirwas MJ. Allergic contact dermatitis to chloroxylenol. Dermatitis 2006;17(3):156-159.

28. Laborde-Castérot H, Villa AF, Rosenberg N, Dupont P, Lee HM, Garnier R. Occupational rhinitis and asthma due to EDTA-containing detergents or disinfectants. Am J Ind Med 2012;55(8):677-682.

29. Matricardi PM, Ronchetti R. Are infections protecting from atopy? Curr Opin Allergy Clin Immunol 2001;1(5):413-419.

30. Strachan DP. Hay fever, hygiene, and household size. BMJ 1989; 299(6710):1259-1260.

31. Yazdanbakhsh M, Kremsner PG, van Ree R. Allergy, parasites, and the hygiene hypothesis. Science 2002;296(5567):490-494. 